\title{
Widespread cutaneous vasculitis associated with diltiazem
}

\author{
R.A. Sheehan-Dare and M.J.D. Goodfield
}

Department of Dermatology, The General Infirmary, Great George Street, Leeds LS1 3EX, UK.

\begin{abstract}
Summary: Two cases of widespread cutaneous vasculitis are described in association with diltiazem, a recently introduced calcium antagonist. In both cases, spontaneous resolution occurred over several weeks following withdrawal of the drug.
\end{abstract}

\section{Introduction}

Diltiazem is a calcium antagonist recently introduced in Great Britain for use in the management of angina pectoris. Skin reactions due to diltiazem are considered to be infrequent. The manufacturers claim a particularly low incidence of side effects. Here we report 2 patients who developed a severe widespread cutaneous vasculitis following oral administration of diltiazem.

\section{Case reports}

Case 1

A woman aged 70 with a 3 month history of angina pectoris was treated with diltiazem $(60 \mathrm{mg}$ twice daily). There was a history of hypothyroidism, atrial fibrillation and left ventricular failure requiring thyroxine, digoxin and bumetanide for 9 years. After 8 days of diltiazem therapy, the patient developed a widespread rash over $24 \mathrm{~h}$. Examination revealed a generalized vasculitic skin eruption consisting of discrete papular erythematous lesions up to $2 \mathrm{~cm}$ in diameter, with a purple discolouration at the centre which failed to blanch completely with pressure. The lesions had become confluent over large areas of the trunk and upper back (Figure 1). There was cardiomegaly and atrial fibrillation, but general examination was otherwise normal. Urinalysis was normal. The white cell count was $11 \times 10^{9} / 1$ and the erythrocyte sedimentation rate (ESR) was $3 \mathrm{~mm} / \mathrm{h}$. Serum antinuclear antibody was not detected. Skin biopsy revealed an inflammatory infiltrate present mainly around and involving dermal capillaries, but also

Correspondence: R.A.Sheehan-Dare,M.B., Ch.B., M.R.C.P. Accepted: 27 January 1988.

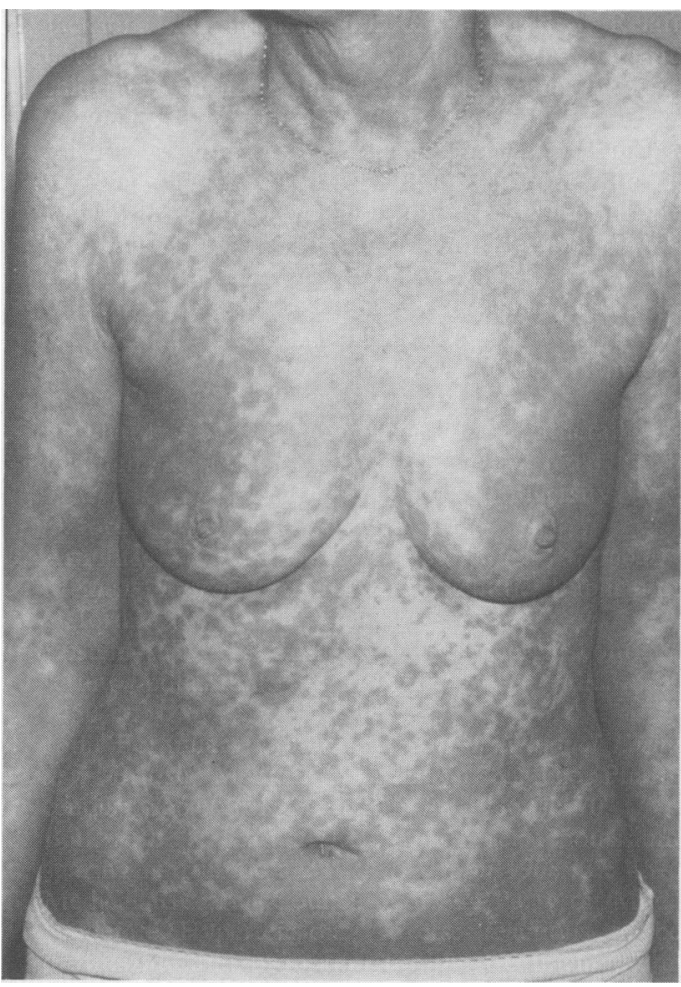

Figure 1 Case 1 at presentation showing a widespread cutaneous vasculitis with confluence of lesions and a central dusky discolouration of most severe lesions.

scattered in the upper dermis. The inflammatory infiltrate consisted predominantly of lymphocytes and histiocytes, but also contained numbers of eosinophils. Extravasation of erythrocytes was also

(C) The Fellowship of Postgraduate Medicine, 1988 
seen. Direct immunofluorescence demonstrated fibrinogen related antigen and $\mathrm{Clq}$ aggregated in the upper dermis and around dermal capillaries. The clinical and histological appearances were of a cutaneous vasculitis.

Diltiazem was withdrawn, but the other drugs were continued. The eruption resolved gradually over 2 weeks with no subsequent recurrence.

\section{Case 2}

A 57 year old woman with angina and ischaemic left ventricular failure was treated with frusemide, amiloride and diltiazem $60 \mathrm{mg}$ twice daily for angina. Eight days later she developed a generalized pruritic eruption of sudden onset associated with general malaise, headache, anorexia and epigastric pain. Examination revealed discrete papular erythematous lesions up to $2 \mathrm{~cm}$ in diameter with a central purple discolouration and varying degrees or purpura. Many of the lesions had become confluent, and small erosions and pustules were seen in some of these areas. The appearances were clinically very similar to case 1 . There was a mild conjunctivitis and oral mucositis, but no frank ulceration. Tender cervical lymphadenopathy was present. There was peripheral oedema due to the severity of the cutaneous inflammation, but the remainder of the general examination was unremarkable. The white cell count was $21.7 \times 10^{9} / 1$ (neutrophil leucocytosis), and the ESR was $30 \mathrm{~mm} / \mathrm{h}$. Serum antinuclear antibody was detected in a titre of 1 in 10. A diagnosis of a drug induced cutaneous vasculitis was made.

All medication was stopped. The skin eruption resolved gradually over 3 weeks. Subsequent rechallenge with oral frusemide and amiloride produced no reaction.

\section{Discussion}

The nature of the eruptions, their time course of onset, the histological and immunofluorescence findings in case 1 and the resolution after withdrawal of diltiazem make a diagnosis of cutaneous vasculitis due to this drug secure in these cases. Cutaneous adverse reactions are said to occur in $1 \%$ of patients treated with this drug. ${ }^{1}$ Most are minor and include photosensitivity, urticaria,

\section{References}

1. McEvoy, G.K. (ed) American Hospital Formulary Service. Drug Information. American Society of Hospital Pharmacists Bethesda, 1987, pp. 727-729.

2. Baker, H. Drug eruptions. In: Rook, A., Wilkinson, D.S., Ebling, F.J.G., Champion, R.H. \& Burton, J.L. (eds) Textbook of Dermatology. Blackwell Scientific Publications, Oxford, 1986, 1239-1279. pruritus and petechiae. $^{1}$ Occasionally isolated reports of more severe reactions such as exfoliative dermatitis and toxic epidermal necrolysis have been reported to the Committee on Safety of Medicines (CSM - personal communication). The two cases reported here were both severe, one having constitutional upset and a degree of mucosal involvement, and were probably due to an immune 'complex mediated hypersensitivity to diltiazem. No previous similar cases have been reported. Of the diltiazem-related cutaneous reactions reported to the CSM, the majority have cited simply 'rash' or 'erythematous rash' (personal communication) and have not indicated the type of eruption.

Although there is at present no satisfactory classification for drug eruptions, a number of specific clinical types can be identified. ${ }^{2}$ The clinical appearances of drug-induced cutaneous vasculitis vary. However, features which usually indicate cutaneous vasculitis include: discrete palpable erythematous lesions up to several centimetres in diameter, often most marked on the legs but which may be widespread and confluent over large areas, a cyanotic area at the centre of individual lesions indicating incipient cutaneous gangrene, failure of blanching on pressure or frank purpura at the centre of individual lesions indicating the presence of extravasated erythrocytes from damaged dermal vessels and areas of necrosis at the centre of individual lesions which may give rise to blistering, pustulation and ulceration. Constitutional upset and mucous membrane involvement are not infrequent. Internal organ involvement may also occur. ${ }^{3}$ Differentiation from other skin reactions such as exanthematic eruptions can be difficult in the mild case. Histology and direct immunofluorescence on appropriate skin biopsy specimens may be helpful in diagnosis. A raised antinuclear antibody titre may indicate druginduced lupus erythematosus in a minority of cases.

When reporting cutaneous adverse reactions to the CSM, an attempt should be made to identify the type of eruption on both clinical and histological grounds so that meaningful conclusions can be drawn from this important source of data.

\section{Acknowledgements}

We would like to thank Dr J.A. Cotterill and Dr N.R. Rowell for their permission to report these cases.

3. Soter, N.A. \& Wolff, S.M. Necrotizing vasculitis. In: Fitzpatrick, T.B., Eisen, A.Z., Wolff, K., Freedberg, I.M., Austen, K.F. (eds) Dermatology in General Medicine. McGraw-Hill, New York, 1987, 1300-1312. 